\title{
Mucinosis eritematosa reticular con buena respuesta al tratamiento tópico
}

\section{Reticular erythematous mucinosis with good response to topical treatment}

\author{
María Belén Elias Kairuz¹, Hassibe Desiree Salich², Jimena Agostina Miranda ${ }^{3}$, Marco Mario \\ Mazzotta $^{4}$, Ivana Laura Forni ${ }^{5}$ y Lorena Andrea Cippitelli ${ }^{6}$
}

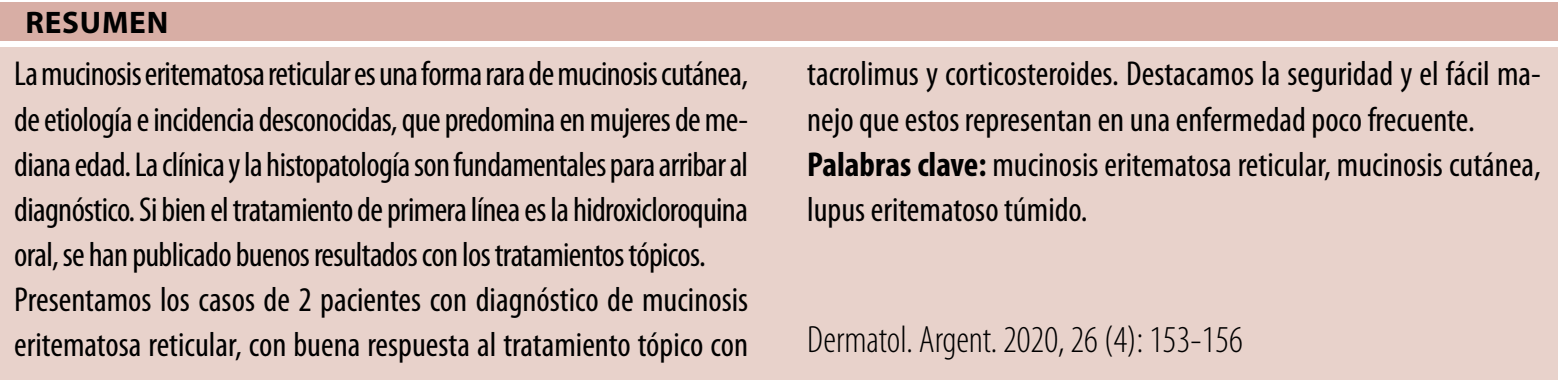

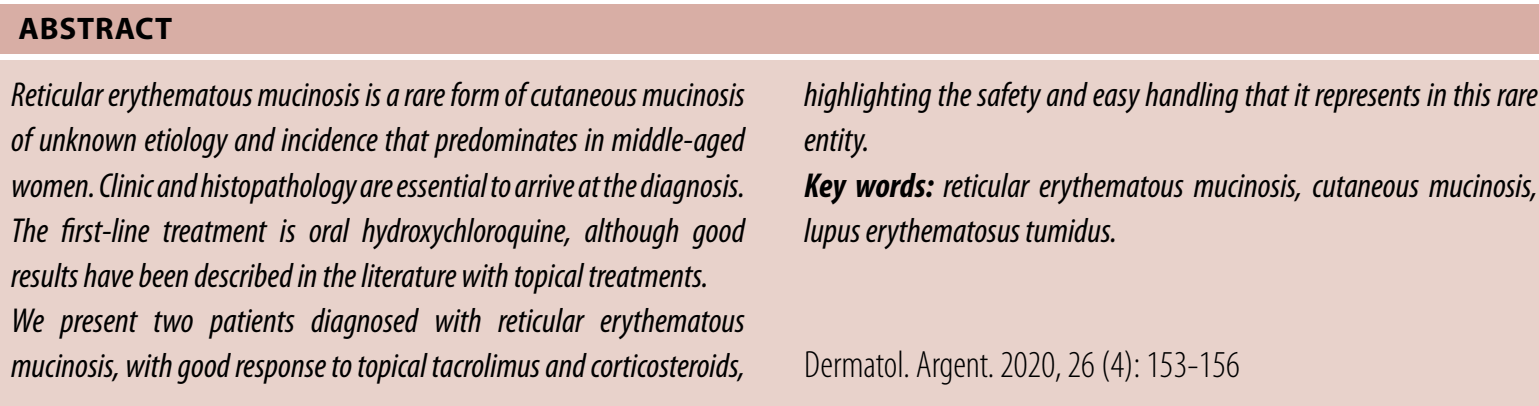

1 Médica Residente de Segundo Año, Posgrado de Clínica Dermatológica

2 Médica Concurrente de Segundo Año, Posgrado de Clínica Dermatológica

${ }^{3}$ Médica Residente de Tercer Año, Posgrado de Clínica Dermatológica

${ }^{4}$ Médico Patólogo. Profesor Adjunto, Cátedra de Anatomía Patológica

${ }^{5}$ Médica. Profesora Adjunta, Cátedra de Clínica Dermatológica

${ }^{6}$ Médica Dermatóloga. Profesora Adjunta, Cátedra de Clínica Dermatológica

Hospital Nacional de Clínicas, Facultad de Ciencias Médicas,

Universidad Nacional de Córdoba, Córdoba, Provincia de Córdoba,

Argentina
Contacto del autor: María Belén Elias Kairuz

E-mail: belenelias416@hotmail.com

Fecha de trabajo recibido: $28 / 10 / 2020$

Fecha de trabajo aceptado: 21/12/2020

Conflicto de interés: los autores declaran que no existe conflicto de interés.

\section{INTRODUCCIÓN}

La mucinosis cutánea forma parte de un grupo heterogéneo de trastornos caracterizados por un depósito de mucina dérmico localizado o difuso. La mucina es un mucopolisacárido producido por fibroblastos, que consiste principalmente en ácido hialurónico unido a heparina y condroitinsulfato. La mucinosis eritematosa reticular (MER) es una forma difusa crónica rara de mucinosis primaria idiopática ${ }^{1}$.
Dado que la etiología exacta no se conoce, el tratamiento suele ser un desafío.

El objetivo de esta publicación es mostrar la buena respuesta de 2 pacientes con MER al tratamiento de segunda línea con tacrolimus y dipropionato de betametasona tópicos, y tenerlo en cuenta como una alternativa segura y de fácil manejo en esta afección poco frecuente. 


\section{SERIE DE CASOS}

\section{Caso clínico 1}

Un paciente de 26 años, con antecedente de diabetes mellitus tipo $1 \mathrm{mal}$ controlada, consultó por presentar una lesión asintomática en la región lumbar izquierda, de 4 meses de evolución. Refería que su coloración se intensificaba ante la exposición solar. En el examen físico se observó una placa eritematosa, de $7 \times 5,5 \mathrm{~cm}$ de diámetro (Foto $1 \mathrm{~A})$, infiltrada a la palpación y con sensibilidad conservada (Foto 1B). Se solicitaron estudios de laboratorio de rutina, función renal, perfil tiroideo, proteinograma, $\mathrm{CPK}$, factor reumatoideo, C3 y C4, ANA, anti-Ro, anti-La, anti-SM, anti-DNA bicatenario, anti-Scl 70, anticentrómero, serología para VIH, VHB, VHC y VDRL, que estuvieron dentro de los parámetros normales, salvo un valor de hemoglobina glucosilada de 8,6\%. Además, se solicitó una ecografía de las partes blandas, que informó: dermis ecogénica y engrosada, ligeramente vascularizada, sin evidencia de lesiones focales. Se realizó una biopsia incisional de la lesión para su estudio anatomopatológico. En ella se observó, en la dermis reticular, un depósito intersticial de mucina y un moderado infiltrado inflamatorio linfocitario superficial y profundo (Foto 2A). Con la tinción de alcian blue se evidenció el depósito de mucina (Foto 2B). Se llegó entonces al diagnóstico clínico-patológico de mucinosis eritematosa reticular. Se instauró como tratamiento tacrolimus ungüento al $0,1 \%$ dos veces por día, con el que se obtuvo, después de 3 meses, una notable reducción de la lesión (Foto 3).

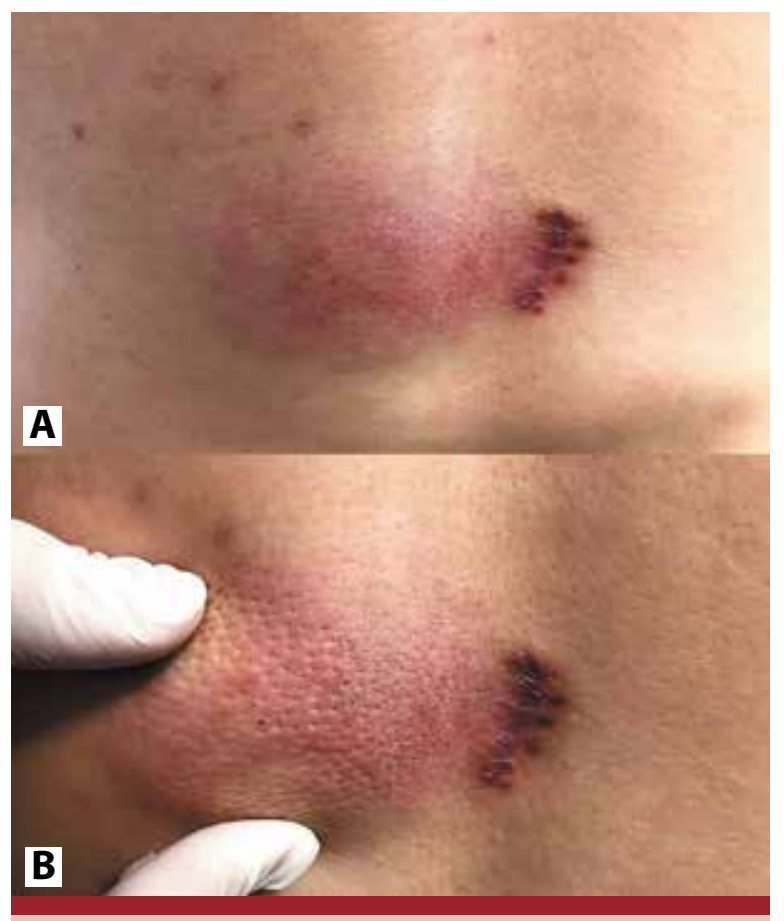

F0T0 1: A) Placa eritematosa de $7 \times 5,5 \mathrm{~cm}$ de diámetro en la región lumbar izquierda. B) Se destaca la infiltración de la placa a la palpación.

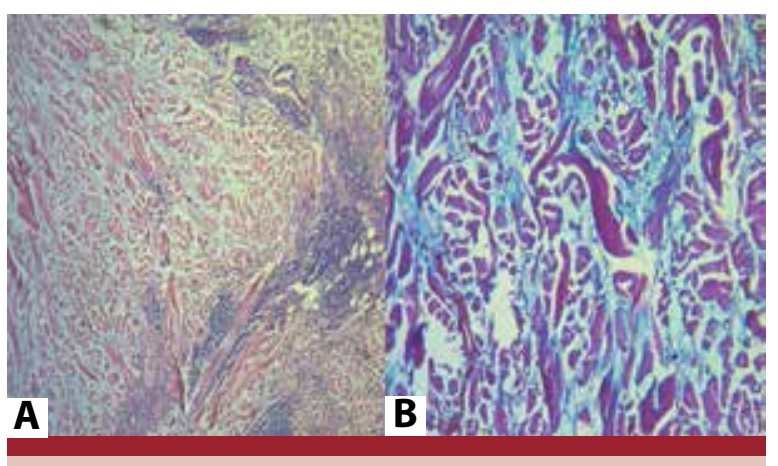

FOTO 2: A) Dermis reticular con depósito intersticial de mucina y moderado infiltrado inflamatorio linfocitario superficial y profundo (HyE, 50X). B) Depósito de mucina (alcian blue, 400X).

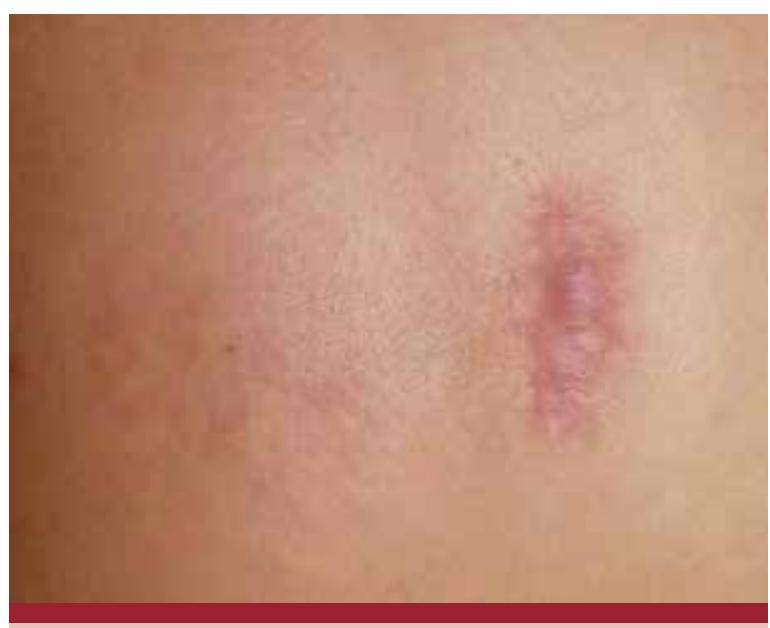

FOT0 3: Caso clínico 1. Placa de mucinosis eritematosa reticular dorsolumbar. Evolución después de 3 meses de tratamiento con tacrolimus ungüento al $0,1 \%$.

\section{Caso clínico 2}

Una paciente de 75 años, con antecedentes personales de enfermedad de Chagas, gastritis, artrosis, dislipidemia e hipertensión arterial en tratamiento, consultó por presentar lesiones cutáneas pruriginosas en el escote, los brazos y las regiones escapular, cervical posterior y lumbar, con distribución bilateral, de aproximadamente 2 años de evolución, sin tratamiento previo. En el examen físico se observaron placas eritematosas de límites irregulares, infiltradas a la palpación, con zonas de excoriación y con sensibilidad conservada (Foto 4). Se solicitaron estudios de laboratorio de rutina, función renal, perfil tiroideo, proteinograma, factor reumatoideo, C3 y C4, ANA, anti-Ro, anti-La, anti-SM, anti-DNA bicatenario y serología para HIV, VHB, VHC y VDRL, cuyos resultados estuvieron dentro de los parámetros normales. Se realizó la biopsia incisional de una lesión del brazo derecho y el análisis anatomopatológico informó: ligera ortoqueratosis y atrofia epidérmica, degeneración vacuolar ba- 
sal y, en la dermis subyacente, un depósito intersticial de mucina difuso, confirmado con la técnica de alcian blue; además, se apreció un leve a moderado infiltrado inflamatorio de tipo mononuclear perivascular superficial y medio. Se arribó al diagnóstico de mucinosis eritematosa reticular. Debido a la situación socioeconómica de la paciente, se decidió iniciar tratamiento con los recursos disponibles en el hospital, que incluyó antihistamínicos por vía oral, emolientes y dipropionato de betametasona crema al $0,05 \%$ dos veces por día, intercalado, en días alternos, con tacrolimus ungüento al $0,1 \%$, dos veces diarias. Las lesiones se resolvieron después de 4 meses de tratamiento, con persistencia de una hiperpigmentación posinflamatoria.

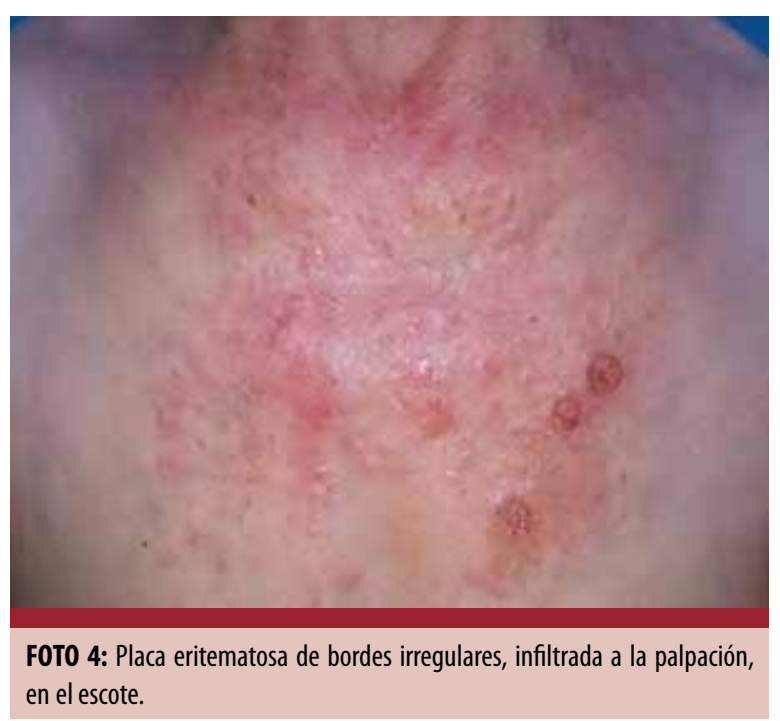

\section{COMENTARIOS}

La mucinosis eritematosa reticular es un trastorno raro, descripto en 1974 por Steigleder et ál. ${ }^{2}$. Esta afección se clasifica actualmente dentro de las mucinosis cutáneas primarias ${ }^{3}$.

Afecta a personas de todas las edades y de ambos sexos, pero predomina en las mujeres jóvenes o de mediana edad ${ }^{4-6}$.

Si bien no se conoce su etiopatogenia, se la asocia a una variedad de afecciones como trastornos de la función tiroidea, diabetes mellitus y cáncer de mama, pulmón y colon. En nuestro trabajo, el paciente del caso 1 era diabético. La infección por HIV, la gammapatía monoclonal y la púrpura trombocitopénica idiopática se describieron en pacientes con MER. Los anticonceptivos orales, la menstruación, el embarazo, el calor, la terapia con rayos $\mathrm{X}$ y la transpiración pueden promover el trastorno o exacerbarlo ${ }^{1,4,6}$.

La exposición al sol casi siempre empeora la erupción, aunque en ocasiones se informó que es benefi- $\operatorname{ciosa}^{4}$. En el paciente del caso 1, la lesión se tornaba fuertemente eritematosa ante la exposición solar.

Desde el punto de vista clínico, la MER se caracteriza por la aparición de máculas o placas eritematosas e infiltradas que, con el tiempo, toman un aspecto reticulopigmentado y, en raras ocasiones, pueden observarse telangiectasias ${ }^{4,7}$. La erupción suele ser asintomática, pero el 30\% de los pacientes refieren prurito ${ }^{5-6}$. Las lesiones se localizan, en general, en la porción central del tórax y la parte alta y central de la espalda y, con menor frecuencia, en la cara, los brazos y el abdomen ${ }^{4-5}$.

Histológicamente, presenta una imagen microscópica característica. La tinción con hematoxilina-eosina revela un leve a moderado infiltrado inflamatorio mononuclear perivascular y perianexial, más intenso en el borde de avance del eritema, el cual yace en la dermis superficial y, a veces, se extiende a la dermis profunda. En algunos casos, se observa una degeneración vacuolar leve de la capa basal ${ }^{5}$. Con la tinción de alcian blue es posible evidenciar el depósito de mucina en la der$\mathrm{mis}^{7,8}$. La separación de los haces de colágeno dérmico es un sello distintivo de la MER ${ }^{4}$.

La entidad presenta un cierto grado de superposición con el lupus eritematoso túmido (LET), ya que comparten una serie de hallazgos comunes que incluyen el predominio femenino, la exacerbación tras la exposición solar, las manifestaciones clínicas, el depósito de mucina, el depósito de IgM en la unión dermoepidérmica $\mathrm{y}$, también, la respuesta a los antipalú$\operatorname{dicos}^{4-5,9,10}$.

Algunos autores consideran que la MER forma parte del espectro de una misma enfermedad, junto con el LET y la infiltración linfocítica de Jessner, ya que tienen características histológicas similares ${ }^{11}$.

La inmunofluorescencia directa (IFD) puede ser positiva tanto en la MER como en el LET. En un estudio comparativo de 11 pacientes con MER y 16 pacientes con LET, Cinotti et ál. encontraron que el $27 \%$ de los primeros y el $42 \%$ de los segundos tenían positividad para depósitos granulares de IgM en la unión dermoepidérmica. También hallaron que el $9 \%$ y el $47 \%$ de los mismos casos de MER y LET, respectivamente, tenían positividad para depósitos granulares de C3 en la unión dermoepidérmica $^{10}$. En los pacientes de los casos analizados, no se realizó la IFD por la falta de especificidad de esta para distinguir esa enfermedades.

No se cuenta con un tratamiento único basado en la evidencia. Se sugieren los antipalúdicos como terapia de primera línea. Producen una mejoría clínica rápida, a menudo dentro de un mes de inicio del tratamiento, pero la recurrencia es común. En general, la hidroxicloroquina, en dosis de 200-400 mg/día, ha 
sido eficaz contra esta afección ${ }^{4,5}$. Los corticosteroides tópicos y sistémicos, el tacrolimus tópico, los antihistamínicos orales, las tetraciclinas, la ciclosporina y la radiación ultravioleta $B$ (UVB) se han utilizado como terapias alternativas, con resultados variables. En nuestro trabajo, el paciente del primer caso presentó una resolución del eritema de más del $90 \%$ tras la aplicación del tacrolimus; en el segundo caso, las lesiones se resolvieron en su totalidad, con hiperpigmentación posinflamatoria luego de la terapéutica con tacrolimus y dipropionato de betametasona alternos. Se han informado buenos resultados con radiación UVA-1, a pesar de la posibilidad de una exacerbación ${ }^{4}$. También

\section{BIBLIOGRAFÍA}

1. Haendchen LC, Sabbag DS, Furlani Wde J, de Souza PK, et ál. Reticular erythematous mucinosis. Cutis 2014;93:22-24.

2. Steigleder GK, Gartmann H, Linker U. REM syndrome: reticular erythematous mucinosis (round-cell erythematosis), a new entity? Br J Dermatol 1974;91:191-199.

3. Del Pozo J, Peña C, Almagro M, Yebra MT, et ál. Systemic lupus erythematosus presenting with a reticular erythematous mucinosis-like condition. Lupus 2000;9:144-146.

4. Thareja S, Paghdal K, Lien MH, Fenske NA. Reticular erythematous mucinosis - a review. Int J Dermatol 2012;51:903-909.

5. Suárez-Amor O, Pérez-Bustillo A, González-Morán MA, Ramírez-Santos $\mathrm{A}$, et ál. Mucinosis eritematosa reticular: respuesta parcial al tratamiento con tacrolimus. Actas Dermosifiliogr 2010;101:105-106.

6. Mansouri P, Farshi S, Nahavandi A, Safaie-Naraghi Z. Pimecrolimus 1 percent cream and pulsed dye laser in treatment of a patient with reticular erythematous mucinosis syndrome. Dermatol Online J 2007;13:22. se informó el uso exitoso del láser de colorante pulsado en 2 pacientes con $\mathrm{MER}^{4,7}$.

En los pacientes jóvenes, las lesiones suelen evolucionar hacia la resolución espontánea con la sustitución del eritema por áreas pigmentadas que desaparecen en meses o ańos ${ }^{7}$. Algunos casos refractarios también pueden desaparecer espontáneamente, incluso después de 15 años ${ }^{4}$.

Se cree que el potente efecto inmunosupresor del tacrolimus sobre las células $\mathrm{T}$, al bloquear la acción de la calcineurina, puede ser el mecanismo a través del cual es efectivo en enfermedades inflamatorias crónicas como la MER 5 .

7. Serrano A, Rodriguez L, Conejo-Mir J. Mucinosis eritematosa reticular. Actas Dermosifiliogr 2004;95:317-319.

8. Braddock SW, Davis CS, Davis RB. Reticular erythematous mucinosis and thrombocytopenic purpura. Report of a case and review of the world literature, including plaquelike cutaneous mucinosis. J Am Acad Dermatol 1988;19:859-868.

9. Rongioletti F, Merlo V, Riva S, Cozzani E, et ál. Reticular erythematous mucinosis: a review of patients's characteristics, associated conditions, therapy and outcome in 25 cases. $\mathrm{Br} J$ Dermatol 2013;169:1207-1211.

10. Cinotti E, MerloV, Kempf W, Carli C, etál. Reticularerythematous mucinosis: histopathological and immunohistochemical features of 25 patients compared with 25 cases of lupus erythematosus tumidus. J Eur Acad Dermatol Venereol 2015;29:689-697.

11. Gruber R, Kuntz T, Oellig F, Paschos A, et ál. Retikuläre erythematöse Muzinose - Sonderform eines kutanen Lupus erythematodes? Z Rheumatol 2020;79:782-784.

* Piense en... DERMATOFIBROSARCOMA PROTUBERANS y elija una opción

Sofía Inés Mathys y Guadalupe Llebaria

División de Dermatología, Hospital de Clínicas José de San Martín, Ciudad Autónoma de Buenos Aires, Argentina

॥ 1

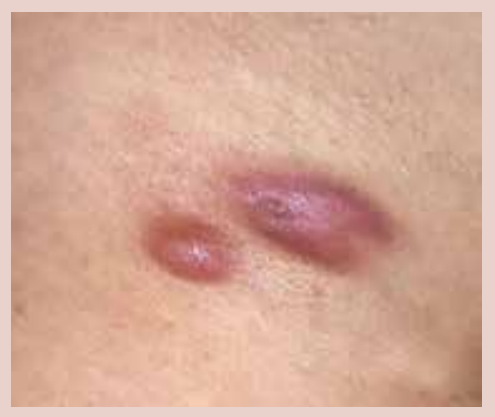

॥ 2

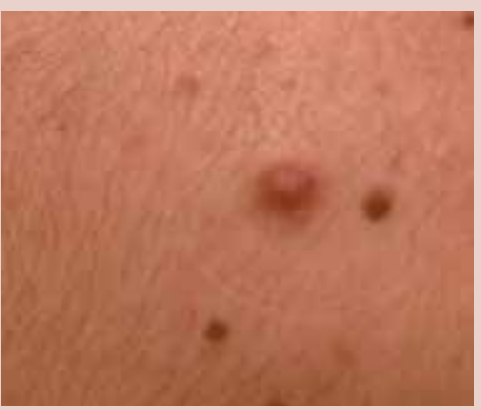

\section{॥ 3}

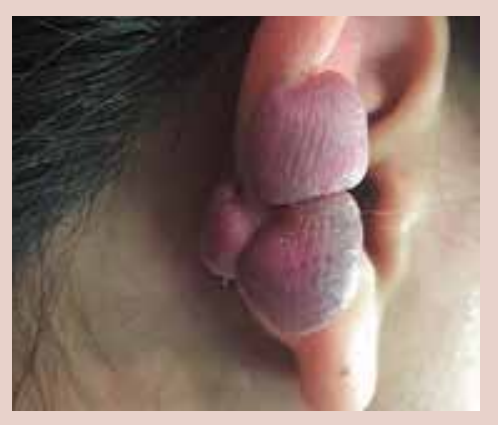

